\title{
Orientation effect in the neutron yield in deuterated Pd target bombarded by deuterium ion beam
}

\author{
O. D. Dalkarov, ${ }^{1}$ M. A. Negodaev, ${ }^{1}$ A. S. Rusetskii, ${ }^{1},{ }^{*}$ A. P. Chubenko, ${ }^{1}$ \\ Yu. L. Pivovarov, ${ }^{2}$ and T. A. Tukhfatullin ${ }^{2}$ \\ ${ }^{1}$ Lebedev Physical Institute RAS (LPI), 53, Leninsky Prospect, 119991 Moscow, Russia \\ ${ }^{2}$ National Research Tomsk Polytechnic University, 30, Lenin Avenue, 634050 Tomsk, Russia
}

(Received 30 November 2018; published 12 March 2019)

\begin{abstract}
At the ion accelerator HELIS at the LPI, the neutron yield is investigated from a deuteron-deuteron (DD) reaction in a deuterated Pd target, during an irradiation of its surface by a $20 \mathrm{keV}$ deuterium (D) ion beam. The measurements of the neutron flux in the D beam direction are performed in dependence on the target angle $\beta$ with respect to the $\mathrm{D}$ beam axis using a multichannel detector based on $\mathrm{He}^{3}$ counters. A significant anisotropy in the neutron yield is observed, and it was higher by a factor of 2 at $\beta=0$ compared to that at $\beta= \pm 30^{\circ}$. The possible reasons for the anisotropy, including $\mathrm{D}$ ion channeling, are discussed. The orientation effect of increasing the relative probability of the DD reaction in Pd due to channeling was investigated by computer simulations using the BCM-2.0 code. The numerically obtained $20 \mathrm{keV}$ D trajectories allow calculating the flux density of channeled D in dependence on the penetration depth and angle of incidence. The enhanced D flux density between crystal planes at a zero incident angle with respect to (200) Pd planes allows a qualitative explanation of the increase of the neutron yield in the DD reaction.
\end{abstract}

DOI: 10.1103/PhysRevAccelBeams.22.034201

\section{INTRODUCTION}

The interactions of the deuterium (D) beam with D-enriched fixed targets are investigated in Refs. [1-5] using the HELIS accelerator facility at the P.N. Lebedev Physical Institute of the Russian Academy of Sciences (LPI). In Ref. [5], the authors investigated the neutron yield in the reaction:

$$
d+d \rightarrow n(2.45 \mathrm{MeV})+{ }^{3} \mathrm{He}(0.8 \mathrm{MeV})
$$

using a textured CVD-diamond target [6] and a $20 \mathrm{keV}$ D beam from the HELIS accelerator which delivers the beam with small angular and energy divergences. In Ref. [5], the authors suggested that the observed enhancement of the neutron yield is connected with both the screening and channeling effects.

The orientation effect of increasing the enhancement factor of a DD reaction in CVD-diamond was investigated by a computer simulation [7]. It was obtained that the flux peaking effect due to channeling up to 2.2 times increases the relative enhancement factor for a parallel beam and up

\footnotetext{
rusets@lebedev.ru
}

Published by the American Physical Society under the terms of the Creative Commons Attribution 4.0 International license. Further distribution of this work must maintain attribution to the author(s) and the published article's title, journal citation, and DOI. to 1.2 times for the $\mathrm{D}$ beam with the angular divergence equaling three critical channeling angles. Qualitative agreement with the experiment in Ref. [5] was obtained.

The results presented in Ref. [5] were confirmed in further experiments at the HELIS accelerator facility. With a smaller step in changing the angle $\beta$, the relative neutron yield at a beam incident angle on a target equal to $\beta=0^{\circ}$ is 5.5 times greater than that at $\beta= \pm 30^{\circ}$; see Fig. 1. In a continuation of these studies, here the CVD-diamond target was replaced by a deuterated Pd target, which allows other screening and channeling conditions.

\section{EXPERIMENTAL METHODS}

The detector and target layouts of the HELIS setup are shown in Fig. 2. The neutron detection efficiencies for the first and second groups of $\mathrm{He}-3$ counters (each group contained 12 counters) were determined using a Cf-252 neutron source placed in place of the target and then used to control the neutron yield.

The relative yield of the DD reaction (1) was determined as $Y_{d d}=n_{n} /\left(S \times I_{d}\right)$, where $n_{n}$ is the longitudinal or transverse neutron flux, $S$ the irradiated area of the target, and $I_{d}$ the $\mathrm{D}$ ion beam current.

We used a deuterated palladium foil target to study the orientation effect in the neutron yield. For comparison, a deuterated titanium target was used. The procedure for preparing the deuterated palladium sample was as follows [8]. The Pd/PdO: $\mathrm{D}_{\mathrm{x}}$ samples of $2.5 \times 1 \mathrm{~cm}^{2}$ in size were prepared by thermal oxidation of a Pd foil (99.95\% purity, 


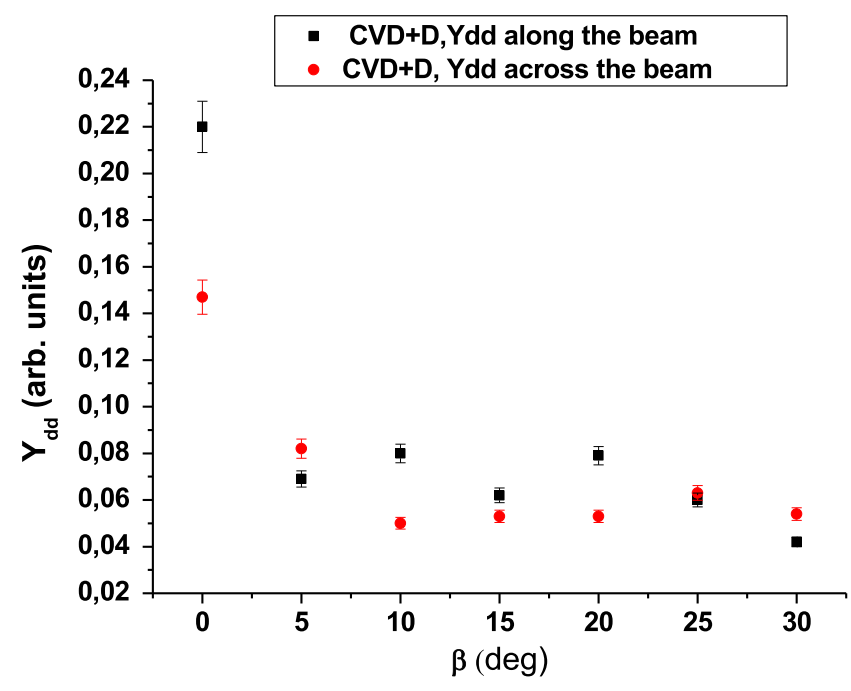

FIG. 1. The neutron yield obtained with the CVD-diamond sample as a function of the angle between the $\mathrm{D}$ beam and the target plane norm, measured in the longitudinal (black squares) and transverse (red diamonds) directions with respect to the $\mathrm{D}$ ion beam. The beam energy is $\mathrm{E}_{d}=21.4 \mathrm{keV}$, and the current is $200 \mu \mathrm{A}$.

$50 \mu \mathrm{m}$ thick). As a result, a $\mathrm{PdO}$ oxide film $\sim 50 \mathrm{~nm}$ thick is formed on the foil surface. Then the samples were saturated with deuterium using electrolysis in a 0.3-M LiOD solution in $\mathrm{D}_{2} \mathrm{O}$ with a $\mathrm{Pt}$ anode at a current density of $20 \mathrm{~mA} / \mathrm{cm}^{2}$ and a temperature of $\sim 290 \mathrm{~K}$ in a cell with separated cathode and anode spaces. After 20-min saturation with deuterium to $x=\mathrm{D} / \mathrm{Pd} \sim 0.73$, the samples were washed in heavy water and cooled by liquid nitrogen to $T=77 \mathrm{~K}$ (sample cooling to liquid nitrogen temperature is necessary to retard deuterium emergence and to study the effect of ionizing radiation on desorption). Then the target was installed into a holder and was placed into an experimental vacuum chamber of the HELIS setup for further study.

The $\mathrm{Ti} / \mathrm{TiO}_{2}: \mathrm{D}_{\mathrm{x}}$ samples $3 \times 1 \mathrm{~cm}^{2}$ in size were made of titanium foil $300 \mu \mathrm{m}$ thick with a $100-150 \mathrm{~nm}$
$\mathrm{TiO}_{2}$ layer by electrolytic saturation with deuterium from a 0.3- $\mathrm{M} \mathrm{D}_{2} \mathrm{SO}_{4}$ solution in $\mathrm{D}_{2} \mathrm{O}$ at a current density of $30 \mathrm{~mA} / \mathrm{cm}^{2}$ in a cell with separated cathode and anode spaces. The samples were used as a cathode (the anode was made of $\mathrm{Pt}$ ). The subsequent weighing showed that on average $0.5 \mathrm{mg}$ of deuterium entered each sample for the electrolysis time of $48 \mathrm{~h}$. This provided the average degree of saturation $x=\mathrm{D} / \mathrm{Ti} \sim 0.1$ at a depth to $1 \mu \mathrm{m}$. The $\mathrm{Ti} / \mathrm{TiO}_{2}: \mathrm{D}_{\mathrm{x}}$ samples remain practically stable at the temperature $T=300 \mathrm{~K}$; they can be saturated by deuterium long before the irradiation.

\section{EXPERIMENTAL RESULTS}

The dependence of the neutron yield from the samples $\mathrm{Pd} / \mathrm{PdO}: \mathrm{D}_{\mathrm{x}}$ and $\mathrm{Ti} / \mathrm{TiO}_{2}: \mathrm{D}_{\mathrm{x}}$ on the angle $\beta$ between the deuteron beam and the normal to the plane of the target is shown in Fig. 3.

A significant anisotropy in the neutron yield is observed for the $\mathrm{Pd} / \mathrm{PdO}: \mathrm{D}_{\mathrm{x}}$ target; it was higher by a factor of 2 at $\beta=0^{\circ}$ compared to that at $\beta= \pm 30^{\circ}$. For samples of deuterated titanium, this effect is not observed.

On the surface of samples of deuterated palladium, pores $100-1000 \mathrm{~nm}$ in size were found, while on the surface of deuterated titanium there is no porous structure (see Fig. 4). The presence of pores on the surface of deuterated palladium can lead to an increase in the effective range of deuterium ions with a normal beam incident on the target and, therefore, an increase in the neutron yield.

\section{SIMULATION OF D TRAJECTORIES AT CHANNELING}

To explain the anisotropy in the neutron yield, we performed the simulations of $\mathrm{D}$ trajectories under the channeling condition in a Pd crystal. Because the energy of $\mathrm{D}$ is $20 \mathrm{keV}$, the parameter $\frac{v}{c}=0.0046 \ll 1$ is small ( $v$ is the deuteron velocity, and $c$ is the light speed); that is, the equation of motion is nonrelativistic. The motion is governed by periodic planar potential $U(x)$, and therefore

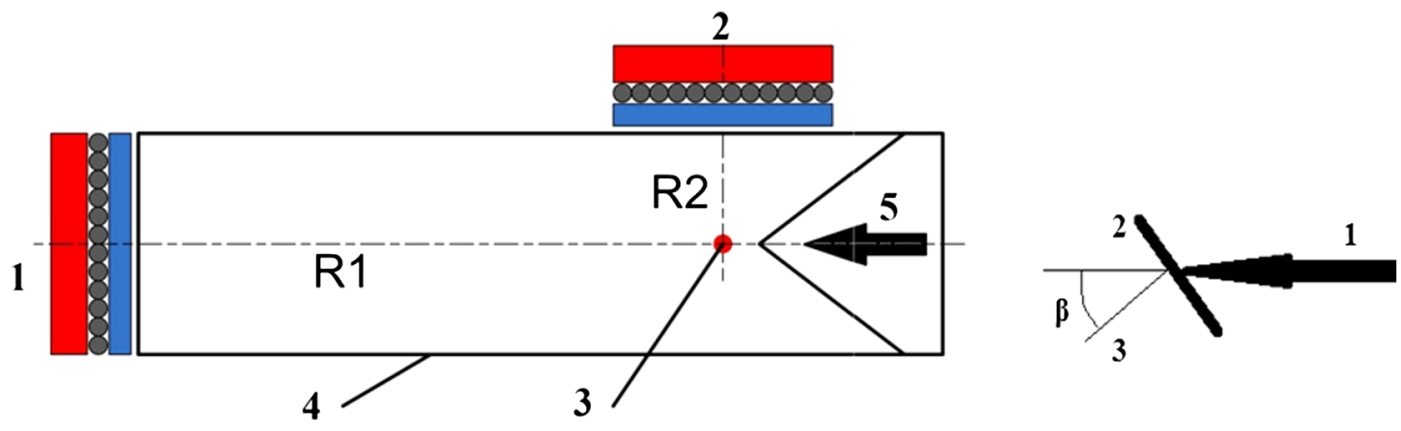

FIG. 2. Left panel: The ${ }^{3} \mathrm{He}$ detector setup at HELIS, representing the first (1) and the second (2) ${ }^{3}$ He-counter groups with radii $\mathrm{R} 1=85 \mathrm{~cm}$ and $\mathrm{R} 2=38 \mathrm{~cm}$, respectively. The target is placed at (3) inside the HELIS beam pipe (4). The ion beam direction is indicated by an arrow (5). Right panel: $\beta$ is an angle between the direction of the beam and the normal to the target plane. The arrow (1) indicates the D beam, (2) is the target, and (3) is the normal to the target surface. 

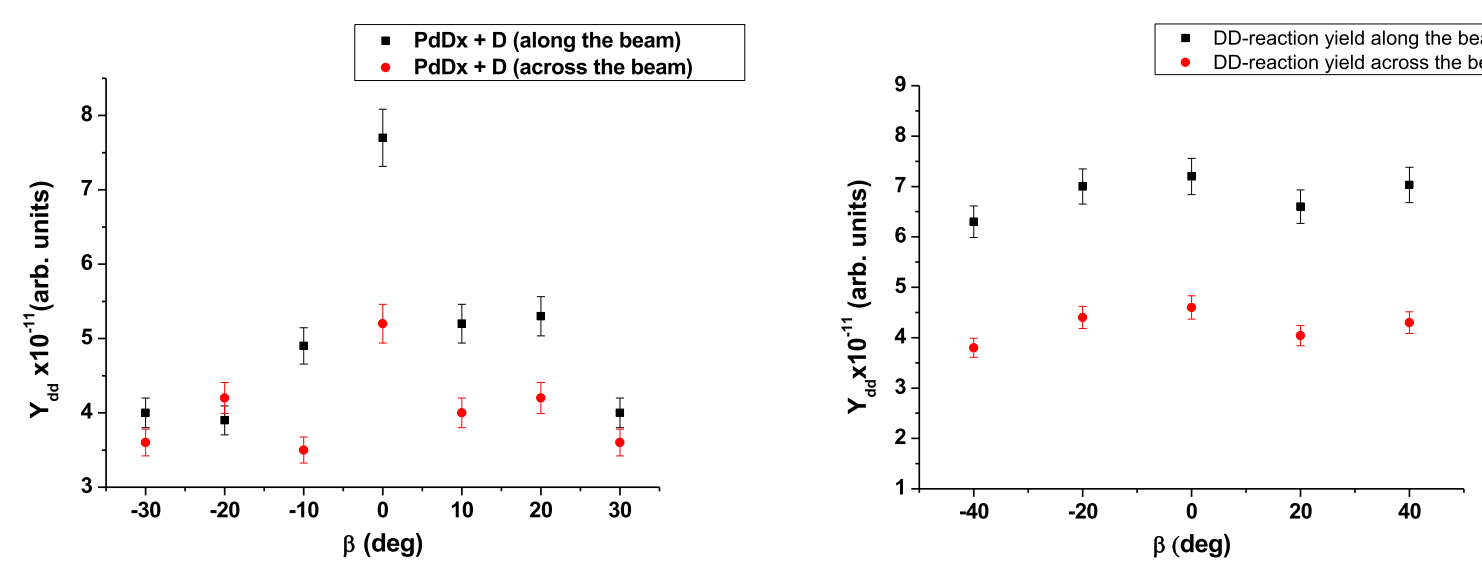

FIG. 3. The dependence of the neutron yield from the samples Pd/PdO: $\mathrm{D}_{\mathrm{x}}$ (left) and Ti/ $\mathrm{TiO}_{2}: \mathrm{D}_{\mathrm{x}}$ (right) on the angle $\beta$ between the deuteron beam and the normal to the plane of the target (black square, along the beam; red circle, across the beam). The energy of the beam $E_{d}=20 \mathrm{keV}$, and the current is $-40 \mu \mathrm{A}$.
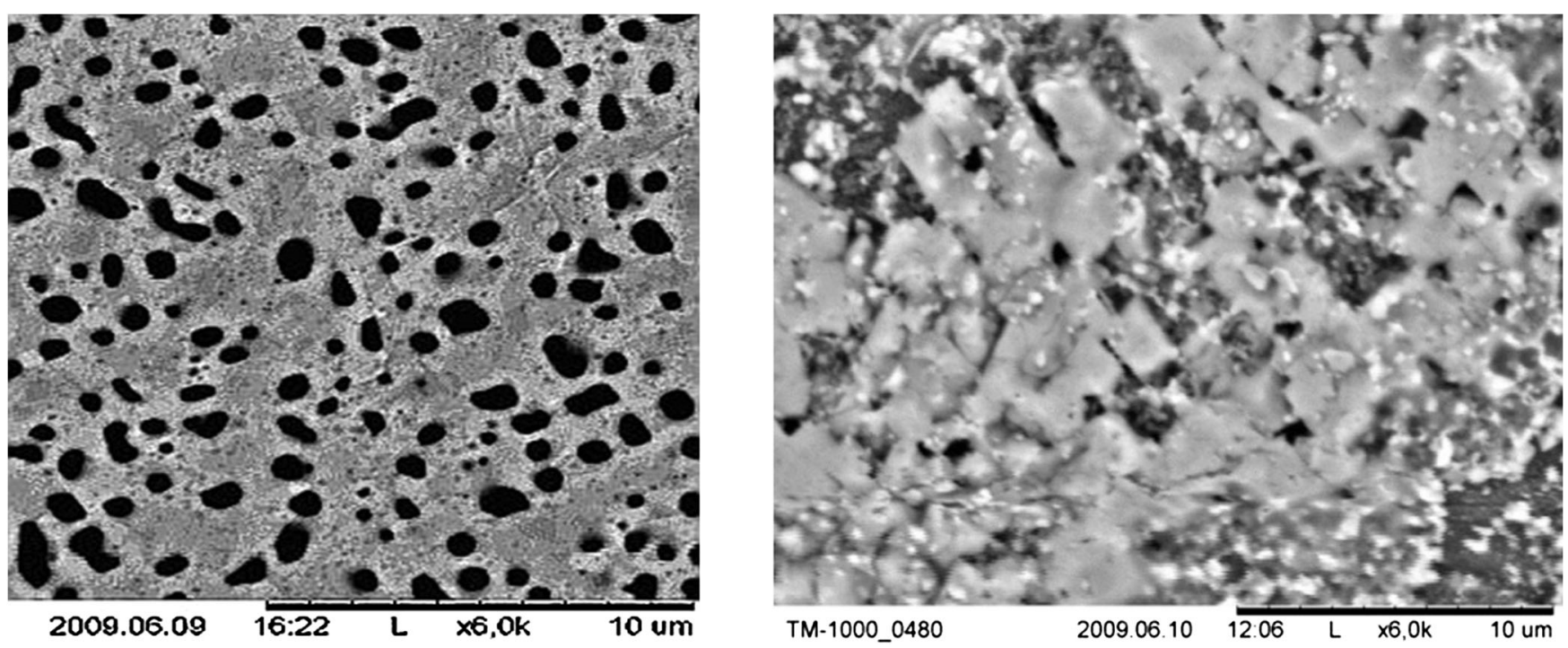

FIG. 4. SEM image $\mathrm{Pd} / \mathrm{PdO}: \mathrm{D}_{\mathrm{x}}$ (left) and $\mathrm{Ti} / \mathrm{TiO}_{2}: \mathrm{D}_{\mathrm{x}}$ (right) after electrolysis and beam irradiation.

$M \frac{d^{2} x}{d t^{2}}=F_{x}=-\frac{\partial U(x)}{\partial x}$

$M \frac{d^{2} y}{d t^{2}}=M \frac{d^{2} z}{d t^{2}}=0 \Rightarrow v_{y}=$ const, $\quad v_{z}=$ const.

If we choose the $Z$ axis parallel to channeling planes and direct it so that $v_{y}(t=0)=0$, then the channeled $\mathrm{D}$ move with constant velocity $v_{z}=$ const along the crystal planes (parallel to the $Z$ axis) and oscillate in the $X$ direction perpendicular to channeling planes.

The numerical solution of the equations of motion (2) was performed using a computer code BCM-2.0 $[9,10]$. Simulations of trajectories take into account angular divergence of the beam (for each entry point, several incident angles $\theta$ are generated) and allow calculating the flux density of the D beam inside the crystal. The calculated flux density is used to analyze the interaction implanted into the crystal $\mathrm{D}$. The incident angle $\theta$ is of the order of the critical channeling angle $\theta_{\mathrm{c}}=\sqrt{\frac{2 U_{0} Z}{M v^{2}}}$ for a nonrelativistic ion and depends on the ion charge $Z e$, depth of the potential well of chosen crystallographic plane $U_{0}$ (depends on crystallographic properties), ion velocity $v$, and mass $M$.

The initial conditions for Eq. (2) include the initial coordinate $x(t=0)=x_{0}$ and velocity $v_{x}(t=0)=v \sin \theta$.

The two typical trajectories of $20 \mathrm{keV} \mathrm{D}$ at (200) channeling in a Pd crystal are shown in Fig. 5 (left panel). Here, (200) are the Miller indices of the planes along which the $\mathrm{D}$ moves at channeling. The impact parameters of the channeled D with implanted D [situated between (200) planes] are shown in the right panel. We suggest that all implanted D are arranged in a perfect Pd lattice 
(a)

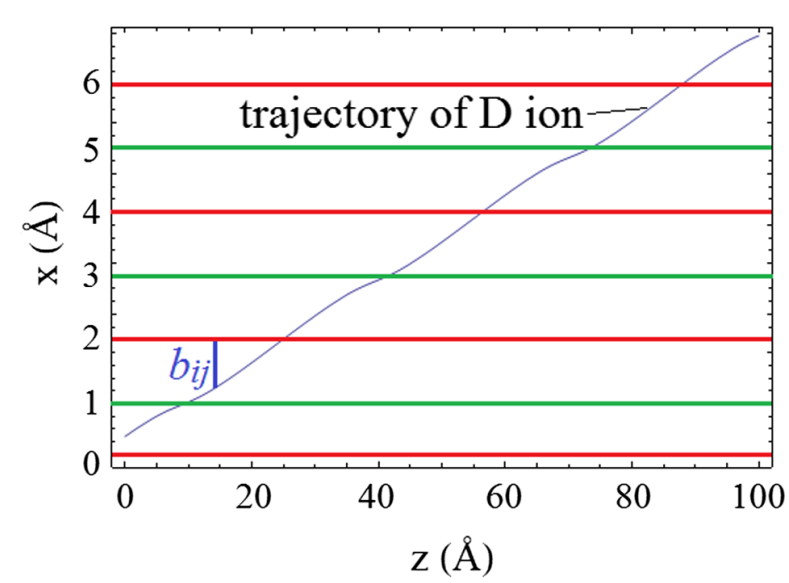

(b)

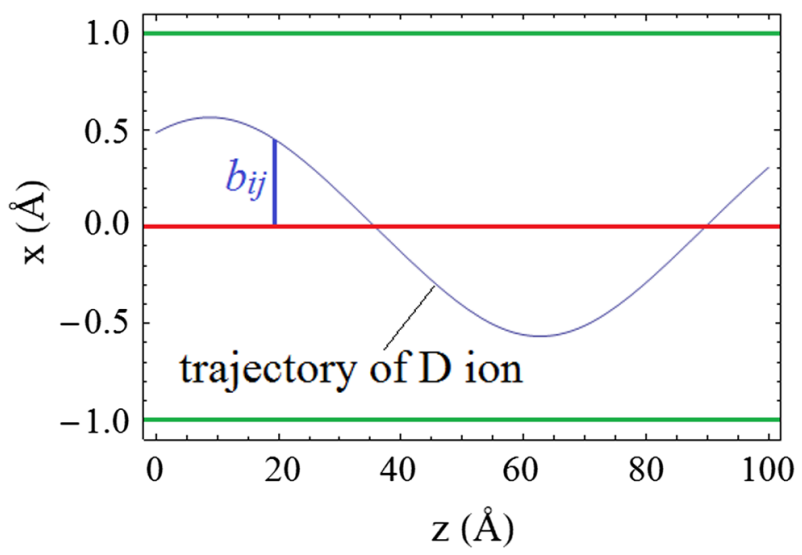

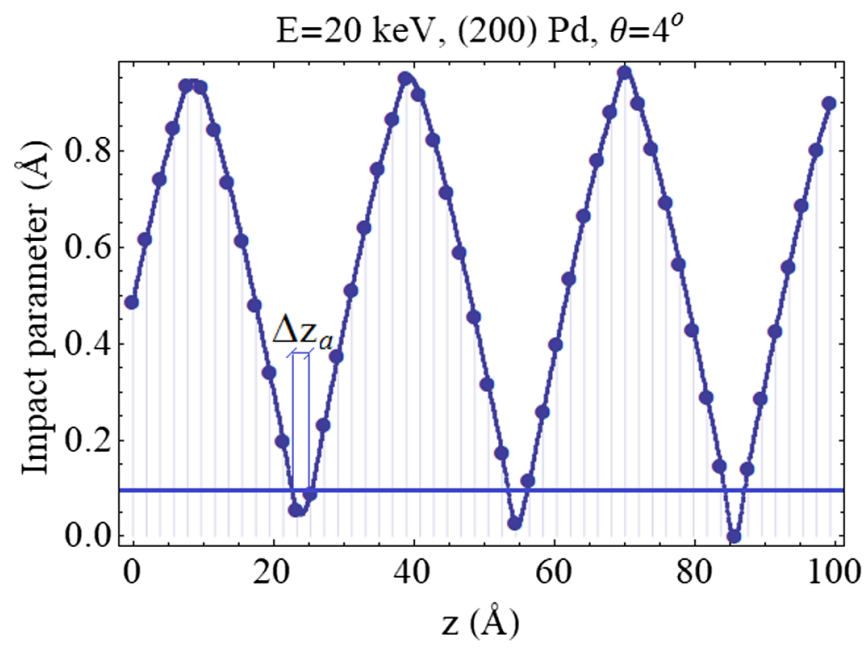

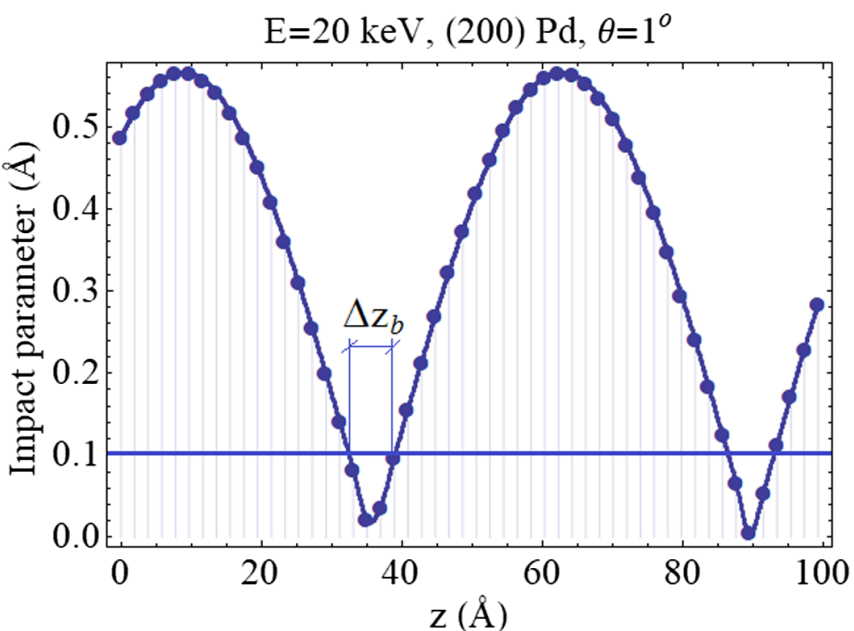

FIG. 5. The typical trajectories of $20 \mathrm{keV}$ D at (200) channeling in a Pd crystal (left panel) and impact parameters of channeled D with a plane formed by implanted D (right panel). (a) The overbarrier motion $\theta>\theta_{c}$ and incident angle $\theta=4^{\circ}$; (b) the underbarrier motion $\theta<\theta_{c}$ (the channeling condition is fulfilled) and incident angle $\theta=1^{\circ}$. The green lines represent schematically (200) $\mathrm{Pd}$ planes, the red lines the planes formed by being implanted into a Pd crystal D (D planes). The dots in the right panels indicate the impact parameters with implanted D along a simulated D trajectory. The connecting curves are drawn only for visualization.

between (200) planes indicated in Fig. 5 by the green lines and form D planes indicated by the red lines.

Consider these trajectories: (a) the overbarrier trajectory crosses D planes three times; (b) the underbarrier trajectory crosses the D plane only two times. One may conclude that in case (a) the reaction yield will be higher compared to case (b). However, the reaction yield depends also on the path $\Delta z$ of $\mathrm{D}$ inside the regions of small impact parameters with implanted $\mathrm{D}$. The path $\Delta z$ inside the $\mathrm{D}$ plane depends approximately on transverse velocity $v_{x}$ as $1 / v_{x}$ and is greater in case (b), i.e., $\Delta z_{b}>\Delta z_{a}$. These regions are shown in the right panels in Fig. 5.

Let us suggest that implanted $\mathrm{D}$ are distributed near the $\mathrm{D}$ plane within $b<0.1 \AA$ (this is of the order of the thermal vibration amplitude of atoms in a crystal lattice). Thus, the path of the (a)-type trajectory inside those three regions is $3 \times \Delta z_{a}=3 \times 3 \AA=9 \AA$, while the path of the (b) trajectory inside those two regions is
$2 \times \Delta z_{b}=2 \times 7.5 \AA=15 \AA$, which means in case (b) the reaction yield should be higher.

This is as a simple as possible explanation of the role of channeling. More exactly, as follows from simulations, the impact parameter is $b_{i j}<0.1 \AA\left(b_{i j}\right.$, impact parameter with implanted D) with seven implanted D [see Fig. 5(b)], while for the above barrier motion $b_{i j}<0.1 \AA$ only with four implanted D. Again, one can say that channeling should lead to the enhancement of the DD reaction yield.

In reality, we deal with the very large number of different trajectories, and to characterize qualitatively the channeling influence (focusing of incident $\mathrm{D}$ beam on implanted $\mathrm{D}$ ), let us introduce the averaged over all simulated trajectories impact parameter

$$
\langle b\rangle=\frac{\sum_{i=1}^{N_{\mathrm{tr}}} \sum_{j=1}^{N_{D}} b_{i j}}{N_{\mathrm{tr}} N_{D}} .
$$




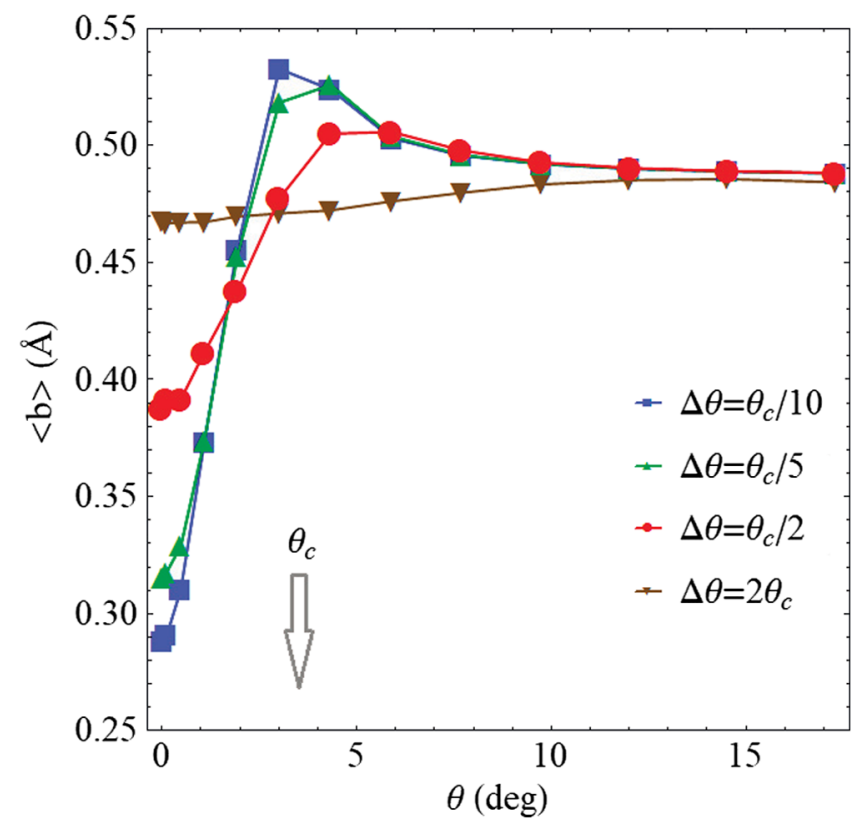

FIG. 6. Simulation results for $20 \mathrm{keV}$ D channeled in a (200) Pd crystal: $b$ versus incident angle $\theta$ for different angular divergences of the incident beam. The critical channeling angle $\theta_{c}=3.58^{\circ}$ is indicated by an arrow.

In Eq. (3), $N_{\text {tr }}$ is the number of deuteron trajectories, and $N_{D}$ is the number of implanted D close to which the trajectory passes. The averaged impact parameter $b$ is shown in Fig. 6 as a function of the incident angle $\theta$ and for different angular divergences of the $\mathrm{D}$ beam. The $b$ value increases with an increase of incident angle $\theta$, because the number of $\mathrm{D}$ involved in channeling motion decreases. The lower the $b$, the greater the DD reaction yield, and vice versa. In particular, the $b$ value is increased by 1.7 times following an increase in the incident angle $\theta$ from zero to $17^{\circ}$ for a $\mathrm{D}$ beam with angular divergence $\Delta \theta=0.1 \theta_{C}=0.358^{\circ}$. This leads to the decrease of the yield of the DD reaction.

Also we calculated the fraction of trajectories for which the impact parameters with $\mathrm{D}$ located within $\mathrm{D}$ planes are less than two radii of $\mathrm{D}$ nucleus $2 R_{D}=3.3 \times 10^{-5} \AA$ :

$$
f\left(b_{i j}<2 R_{D}\right)=\frac{N_{\mathrm{tr}}\left(b_{i j}<2 R_{D}\right)}{N_{\mathrm{tr}}}
$$

This value drops up to 2 times with an increase of the incident angle $\theta$ (see Fig. 7). That is, again the qualitative indication why the DD reaction yield increases due to channeling. Besides, the role of incident beam angular divergence is clearly seen.

Figures 6 and 7 demonstrate also the sensitivity of the orientation effect to the incident $\mathrm{D}$ beam angular divergence $\Delta \theta$. To comment, the critical channeling angle for $E=20 \mathrm{keV}$ D channeled in a (200) C crystal is equal to $1.39^{\circ}$, while for $E=20 \mathrm{keV}$ D ion channeled in a (200) Ti

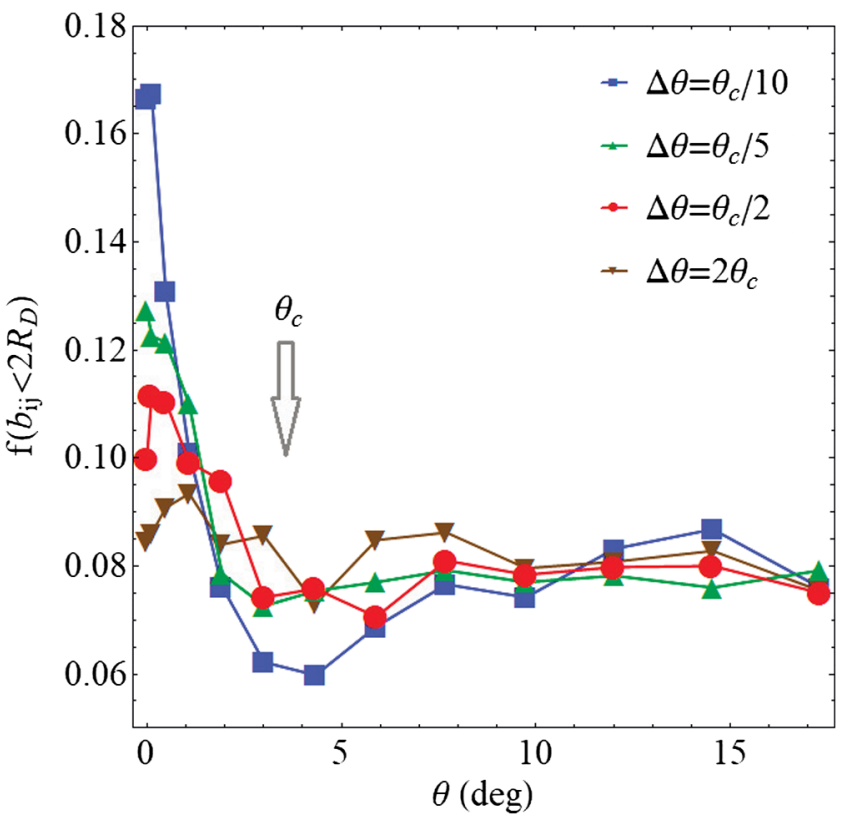

FIG. 7. Simulation results for $20 \mathrm{keV}$ D channeled in a (200) Pd crystal: $f\left(b_{i j}<2 R_{D}\right)$ versus incident angle $\theta$ for different angular divergences of the incident beam. The critical channeling angle $\theta_{c}=3.58^{\circ}$ is indicated by an arrow.

crystal it is equal to $2.44^{\circ}$. That means at an equal beam angular divergence the orientation effect should manifest itself more brilliantly when the Pd target is used, if other factors (see Secs. II and III) are excluded. The more detailed simulations should include more information, namely, on the reaction properties (probability, energy dependence, screening effect, etc.).

\section{CONCLUSIONS}

Previous work with diamond showed the presence of an orientation effect at irradiation of a textured polycrystalline diamond target [most crystallites have an orientation of (100)] and its absence when working with a polycrystalline target in which the crystallites are oriented chaotically. When the Ti target is irradiated, the orientation effect is not observed. When the Pd target is irradiated, an orientation effect is also observed, which can be caused by the presence of pores in the direction of the beam or textured polycrystalline structure (it is known that, in cold-rolled foils, crystallites have a predominant orientation). It is observed that the crystalline structure and the orientation of the sample with respect to the beam have an impact on the neutron yield. The highest yield is recorded with the target oriented perpendicular to the beam. Such a strong angular dependence of the neutron yield could indicate the presence of narrow channels in the sample, where the bulk of deuterium, trapped during the electrolysis, is concentrated. These channels could be created in pores on a Pd surface after electrolysis and beam irradiation. Samples without channels on the surface do not show the dependence of the 
neutron yield on the orientation in the ion beam. The presence of channels possibly may cause further focusing of neutrons along the channel direction.

Another reason for the appearance of the orientation effect can be connected with the channeling and so-called flux peaking. The orientation effect of increasing the relative probability of a DD reaction in Pd due to channeling was investigated by computer simulations using the BCM-2.0 code [10]. The numerically obtained $20 \mathrm{keV} \mathrm{D}$ trajectories allow us to calculate the flux density of channeled D in dependence on the penetration depth and angle of incidence with respect to channeling planes. The enhanced D flux density between Pd (200) crystal planes (where implanted D are located) at a zero incident angle with respect to (200) Pd planes allows a qualitative explanation of the increase of the neutron yield in the DD reaction.

The further development of a computer code is in progress. This will include a detailed analysis of impact parameter dependence of DD reaction probability, by conjugating the classical definition of the reaction cross section (integral over impact parameters) from one side, and real yield of DD reaction from another side, which is the convolution of an energy-dependent cross section (includes the astrophysical $\mathrm{S}$ factor) and the effect of screening and energy loss. Other crystal effects like thermal vibrations of implanted D in a Pd lattice and D energy loss will be included, too.

One should comment also on several factors that may influence the $\mathrm{D}$ channeling and also should be taken into account in improvment (further development) of the computer code. The first one is the charge-changing process in the bulk of the target (capture and loss of the target electron), important if the D velocity does not exceed the Bohr velocity, that is, at D energy $\leq 50 \mathrm{keV}$; see, e.g., Ref. [11]. Two others are the multiple scattering (leading to dechanneling) and ionization (electronic) energy loss [12] leading to a decrease in $\mathrm{D}$ velocity and the disappearance of channeling.

In conclusion, although the dependence of the neutron yield on the angle of incidence (orientation effect) can be explained by D channeling in the crystal structure (fluxpeaking effect), this does not explain the difference in the yield of neutrons along and across the direction of the beam (angular anisotropy). Further experimental studies and simulations are necessary.

\section{ACKNOWLEDGMENTS}

This research was supported by Tomsk Polytechnic University CE Program.

[1] A. V. Bagulya et al., Study of DD-reaction yields from a $\mathrm{Pd} / \mathrm{PdO}: \mathrm{D}_{\mathrm{x}}$ heterostructure at low energies using the GELIS setup, Bull. Lebedev Phys. Inst. 39, 247 (2012).

[2] A. V. Bagulya et al., Study of DD-reaction yields from the $\mathrm{Ti} / \mathrm{TiO}_{2}: \mathrm{D}_{x}$ heterostructure at low energies using the GELIS setup, Bull. Lebedev Phys. Inst. 39, 325 (2012).

[3] A. V. Bagulya et al., Enhancement of DD-Reaction yields from the $\mathrm{Pd} / \mathrm{PdO}: D_{x}$ heterostructure by $N^{+}$and $\mathrm{Ne}^{+}$ion beams using the GELIS setup, Bull. Lebedev Phys. Inst. 40, 282 (2013).

[4] A. V. Bagulya et al., Enhancement of DD-reaction yields from the $\mathrm{Ti} / \mathrm{TiO}_{2}: D_{x}$ heterostructure by $\mathrm{H}^{+}$and $\mathrm{N}^{+}$ion beams using the GELIS setup, Bull. Lebedev Phys. Inst. 40, 305 (2013).

[5] A. V. Bagulya et al., Channeling effect in polycrystalline deuterium-saturated CVD diamond target bombarded by deuterium ion beam, Nucl. Instrum. Methods Phys. Res., Sect. B 355, 340 (2015).

[6] V. G. Ralchenko et al., Fracture strengths of optical quality and black polycristalline CVD diamonds, Diam. Relat. Mater. 23, 172 (2012).

[7] A. V. Bagulya, O. D. Dalkarov, M. A. Negodaev, Yu. L. Pivovarov, A. S. Rusetskii, and T. A. Tukhfatullin, Orientation effect in $d(d, n)^{3} \mathrm{He}$ reaction initiated by $20 \mathrm{keV}$ deuterons at channeling in textured CVD-Diamond target, Nucl. Instrum. Methods Phys. Res., Sect. B 402, 243 (2017).

[8] I. P. Chernov et al., Nuclear reactions in the $\mathrm{Pd} / \mathrm{PdO}: D_{x}$ and $\mathrm{Ti} / \mathrm{TiO}_{2}: D_{x}$ systems excited by ionizing radiation, J. Exp. Theor. Phys. 112, 952 (2011).

[9] Y. Takabayashi et al., Angular distributions of relativistic electrons under channeling in half-wavelength crystal and corresponding radiation, Nucl. Instrum. Methods Phys. Res., Sect. B 355, 188 (2015).

[10] S. V. Abdrashitov et al., BCM-2.0-The new version of computer code "Basic Channeling with Mathematica(C) Nucl. Instrum. Methods Phys. Res., Sect. B 402, 106 (2017).

[11] I. A. Baranov, Yu. V. Martynenko, S. O. Tsepelevich, and Yu. N. Yavlinskiu, Inelastic sputtering of solids by ions, Sov. Phys. Usp. 31, 1015 (1988).

[12] P. Sigmund, Particle Penetration and Radiation Effects (Springer, Heidelberg, 2014), Vol. 2. 